\title{
Quelques généralités sur les sécheresses et leur prévision
}

PAR

\section{G. Oberlin}

Chef de la division Hydrologie du C. T. G. R. E. F.

(Centre Terhnique du Génie Rural, des Eaux et des Forêts)

A l'occasion de la sécheresse qui sévit sur une partie de la France en cette année 1976, il nous a semblé utile de formuler quelques généralités sur les possibilités de prévision de ces phénomènes. Cette note se veut très générale et ne prétend pas décrire des méthodes possibles de prévisions. Elle ne contient donc ni exposé technique, ni bibliographie.

Le terme général de sécheresse recouvre diverses acceptions et peut concerner différentes réserves d'eau. Il est utile de bien les individualiser car elles conduisent à des gestions de pénuries assez différentes et, surtout, à des délais de prévision fort éloignés les uns des autres.

Au terme de sécheresse nous donnerons un sens plutôt opérationnel : non seulement déficit par rapport à la situation moyenne, mais encore gêne importante pour les usagers. Certains déficits ne sont en effet pas systématiquement gênants : sol sec à l'automne, débits modestes en hiver, etc. .

Nous distinguerons quatre réserves dans ce qui suit: l'air, le sol, les cours d'eau (lacs, et donc réservoirs de barrage, inclus) et le sous-sol (nappes).

\section{Définitions proposées}

\subsection{Sécheresse atmosphérique : (S. Atm.)}

Il ne pleut pas et l'air est éventuellement sec de surcroît. Elle peut se produire à toute période de l'année, mais n'est réellement gênante que lorsque les températures sont également élevées. Ses effets à court terme sont limités car la végétation, si elle est incommodée, réagit par ré- gulation stomatique ce qui, tout au plus, réduit un peu la production végétale.

\subsection{Sécheresse du sol : (S. Sol)}

C'est la sécheresse agricole classique. Elle est surtout gênante au printemps et en été, assez peu en automne et en hiver où elle est d'ailleurs rare.

\subsection{Sécheresse des cours d'eau : (S. Cou.)}

Il s'agit d'une faiblesse des débits des rivières, qui sont à l'étiage, et donc des réservoirs naturels ou artificiels situés sur leurs cours. En fait, il y a des décalages dans le temps pour ces derniers, décalages qui les rapprochent un peu (pour les plus largement dimensionnés d'entre eux) des nappes citées au § 4.4. Cette sécheresse pose des problèmes de pollutions (dilution réduite), met en péril la vie biologique du cours d'eau, gêne ou empêche des pompages ou dérivations.

\subsection{Sécheresse souterraine : (S. Sou)}

Il s'agit d'un niveau anormalement bas de nappes. Certains forages ou puits s'assèchent ou ne peuvent plus délivrer leur débit d'exploitation habituel.

Toutes ces définitions seraient à nuancer et, dans une application locale, il faudrait préciser en fonction des caractéristiques de ces différentes réserves : sols épais ou minces, cours d'eau permanents rares ou nombreux. lacs profonds ou petits, nappes de surface ou profondes. etc. . . 


\section{Interaction entre ces différentes sécheresses}

Nous supposons d'abord des conditions naturelles, c'est-à-dire sans effets de pompage ou de prélèvement.

Les S. Atm. sont évidemment à l'origine de toutes les autres, sauf circonstances très locales et exceptionnelles. Cependant, leur influence sur les autres est très variable à cause du caractère saisonnier du phénomène d'évapotranspiration (ETR) : presque nulle en hiver, cette ETR est capable de prélever plus de $100 \mathrm{~mm}\left({ }^{1}\right)$ certains mois d'été, soit l'équivalent d'un mois exceptionnellement pluvieux $\left({ }^{1}\right)$.

\subsection{Influences sur les S. Atm.}

L'effet de retour des autres sécheresses sur les S. Atm. est faible. Tout au plus pouvons-nous noter une légère influence des $S$. Sol sévères et de grande étendue, susceptibles de prolonger ou d'aggraver un peu une S. Atm. déjà existante.

Inversement, un sol très humide et de grande étendue peut localement atténuer une S. Atm., mais certainement pas la supprimer.

\subsection{Influences sur les S. Sol}

Les S. Sol sont causées par des S. Atm. de durée vàriable selon les saisons et les réserves en eau initiales $\mathrm{du}$ sol. Nous pouvons avancer les ordres de grandeur suivants :

- minimum de 2 à 3 semaines l'été

- minimum de 1 à 2 mois au printemps (davantage si I'hiver a été pluvieux)

- minimum de 1 à 2 mois à l'automne, et encore sous réserve que la $\mathrm{S}$. Sol soit amorcée dès la fin de l'été

- en hiver, une S. Sol est quasi-impossible ; même si elle existe à la fin de l'automne (rare), la simple rosée (fréquente et abondante si $\mathrm{S}$. Atm.) suffit à réhumidifier le sol, légèrement mais suffisamment compte tenu des besoins faibles de cette saison.

Un climat pluvieux fait disparaître une S. Sol :

- immédiatement en hiver

- assez rapidement au printemps : quelques jours ou quelques dizaines de $\mathrm{mm}$ de pluie

- lentement en été : il faut un temps très pluvieux ou des pluies réellement fréquentes s'étendant sur de nombreux jours

- assez rapidement en automne.

\subsection{Influence sur les S. Cou.}

Les S. Cou. sont dues, soit à des S. Atm. un peu prolongées, soit à des S. Sol., soit à des S. Sou. Nous éliminerons les $\mathrm{S}$. Sol. car elles sont assez directement reliées aux S. Atm.

(1) Ordre de grandeur d'une situation de plaine en climat français moyen.

\subsubsection{Influence des S. Atm. sur les S. Cou.}

Elle n'est nette qu'en automne et en hiver: des S. Atm. en ces saisons empêchent les débits d'augmenter (pas de crues).

Au printemps, il faut que la S. Atm. soit sévère et prolongée pour qu'une $\mathrm{S}$. Cou. apparaisse ; par contre, une S. Cou. existant à la sortie de l'hiver (rare) est évidemment maintenue et progressivement aggravée par une S. Atm. de printemps.

En été, les débits baissent presque toujours, qu'il y ait ou non une S. Atm. ; cette dernière ne peut que précipiter la naissance ou l'aggravation de la S. Cou. habituelle (étiage normal).

Des climats pluvieux font disparaître les S. Cou. :

- rapidement en hiver

- assez lentement au printemps

- très lentement, et souvent pas du tout, en été

- assez rapidement en automne.

\subsubsection{Influence des S. Sou. sur les S. Cou.}

Seules les S. Sou. des nappes plus ou moins superficielles sont ici concernées.

Pour un grand nombre de cours d'eau, les S. Sou. sont à l'origine des S. Cou. pendant les périodes d'étiage :

- de l'été, sauf climat très pluvieux

- d'automne, si une S. Atm. règne

- d'hiver, si une S. Cou. amorcée en automne se prolonge, suite à une $\mathrm{S}$. Atm. persistante.

Inversement, des nappes abondantes produisent des étiages élevés, mais évidemment pas de crues.

En dehors des périodes d'étiage, l'influence d'une S. Sou. est faible sur les débits, sauf régime particulier (bassin de craie, par exemple)

\subsection{Influence sur les S. Sou.}

En première approximation, seules des S. Atm. d'hiver sont susceptibles de générer des S. Sou. Des S. Atm. prolongées de printemps peuvent encore aggraver une S. Sou. ou la rendre naissante, si l'hiver a été modérément pluvieux. Des $\mathrm{S}$. Atm. d'automne retardent le moment où les nappes se rechargent.

Inversement, seuls des hivers pluvieux sont susceptibles de faire disparaître des S. Sou., éventuellement aussi des printemps très pluvieux qui suivent des hivers pluvieux (prolongation de l'infiltration profonde), ou encore des automnes exceptionnellement humides ("pré-hiver").

Des S. Atm. d'été ou des étés très pluvieux n'ont pratiquement pas d'influence sur les $\mathrm{S}$. Sou.

Les S. Sou. peuvent se cumuler d'année en année et n’ont donc généralement pas de régulation annuelle.

Comme cela a été répété, les ordres de grandeur des temps de réponse précédents sont à nuancer localement, en particulier pour les nappes qui réagissent aux séquences pluvieuses avec des délais variant de quelques jours (nappes superficielles) à quelques mois (nappes "ordinaires" envisagées ci-dessus), voire quelques années (nappes captives). 


\section{Modifications de ces interactions dues à l'utilisation des équipements hydrauliques}

Ce qui précède est aussi à nuancer en fonction des équipements hydrauliques locaux : barrages-réservoirs, stations de pompage, etc...

\subsection{Cas idéal : équipement diversifié et de capacité suffi- sante}

Dans ce cas, le système pourrait être entièrement régulé, sous réserve d'une gestion bien organisée et surtout respectée. Les considérations du $\S 2$ n'auraient alors plus de valeur, sauf pour les S. Atm.

Par exemple, dès qu'une S. Sol. apparaịtrait, l'irrigation viendrait l'annuler; ou encore, dès qu'une S. Cou. apparaîtrait, une lâchure de réservoir de grande capacité (régulation interannuelle), ou un arrêt de prélèvement (compensé par un pompage accru de nappe) l'annulerait.

\subsection{Cas réel : équipements limités $\left({ }^{2}\right)$}

La régulation ne pourra plus se faire, vis à vis du temps, au-delà d'un certain seuil de sécheresse ; c'est-à-dire qu'il y aura tout de même des alternances de sécheresse (plus ou moins réduites par rapport aux naturelles, selon les capacités des équipements) et d'abondance, mais il pourra y avoir une péréquation entre les types de sécheresse. Par exemple, l'irrigation atténuera une S. Sol., mais aggravera une S. Cou. ou, indirectement, une S. Sou.

\subsection{Rôles des nappes vis à vis des S. Sol. et S. Cou.}

En cas de sécheresse sévère, affectant par exemple les 3 premiers types (S. Atm., S. Sol. et S. Cou.), il peut tout de même y avoir de fortes réserves d'eau dans les nappes, en particulier profondes, même si celles-ci sont par ailleurs affectées d'une S. Sou.

Il est alors tentant de les utiliser, dans la plupart des cas indirectement $\left({ }^{3}\right)$, pour atténuer la $\mathrm{S}$. Sol., voire même la $\mathrm{S}$. Cou (débit réservé, adduction d'eau, etc...), quitte à créer ou aggraver la $\mathrm{S}$. Sou. Ce palliatif est d'ailleurs si tentant qu'il est souvent utilisé de manière sauvage. Nous pensons qu'il pourrait se justifier, mais sous réserve de satisfaire, entre autres, les conditions suivantes:

- avoir bien hiérarchisé les besoins et ne pas utiliser des eaux pures (nappes profondes) pour satisfaire directement des besoins pouvant se contenter d'eaux de

(2) Rappelons qu'un équipement de régulation des ressources en eau est en principe dimensionné à l'aide d'une méthode de type "coût-avantage", c'est-à-dire qu'il ne régule que pour des besoins définis et jusqu'à une certaine fréquence.

(3) Cas de plus en plus fréquent des systèmes mixtes d'adduction d'eau (forage + prélèvement d'eau de surface) qui peuvent, si les équipements le permettent, provisoirement abandonner les prélèvements et ne compter que sur les seuls forages. moindre qualité (irrigation) ; ceci exige un équipement diversifié avec une certaine interconnection des réseaux;

- avoir étudié les conséquences hydrogéologiques : intrusion d'eaux marines, polluées, éventuelle subsidence, etc...

- disposer des équipements nécessaires : (forages profonds, pompes de capacité suffisante, etc. .)

- enfin, et surtout, disposer d'une règlementation opérationnelle complexe étendant ses contraintes sur une période pluriannuelle, de manière à permettre à la nappe sollicitée d'éliminer sa $\mathbf{S}$. Sou dans les années humides qui suivront le pompage exceptionnel.

Les bénéficiaires de ce secours devront donc, une fois les années humides revenues, réduire leur pompage pendant une certaine durée ou mettre en ouvre, si les conditions locales s'y prêtent, les délicates techniques de réalimentation de nappes. Comme leurs besoins ne peuvent probablement être beaucoup réduits, il leur faudra, pendant cette période, prélever dans les cours d'eau et leurs réservoirs.

Nous pouvons donc conclure qu'une politique de prélèvements exceptionnels des ressources en eau profonde, utile en cas de pénurie des eaux de surface, n'exclut d'aucune manière une politique concomitante de mise en réserve des eaux de surface : forages et barrages $\left({ }^{4}\right)$ doivent être menés de façon coordonnée et des règles de gestion à échéances pluriannuelles doivent être élaborées et respectées.

En corollaire, nous retrouvons la nécessité de disposer, pour toute unité hydraulique (bassin versant, bassin sédimentaire, etc...), d'une instance régionale de compétence générale en matière de ressources en eaux, capable d'assurer une coordination suffisante entre les divers usagers et services. Dans ces conditions, des règlementations aussj délicates que celles citées plus haut pourraient être considérées comme applicables, et l'atténuation des sécheresses superficielles grâce aux ressources profondes pourrait devenir une réalité, sans que soit détruit le patrimoine que représentent ces réserves profondes.

\section{Conséquences sur les délais de prévision}

Nous raisonnerons en conditions naturelles et moyennes pour la France. Nous donnerons un ordre de grandeur des délais maximaux qu'il est raisonnable d'avancer.

\subsection{Prévision des S. Atm.}

Une S. Atm. est imprévisible au-delà de quelques jours : 1 à 2 en temps ordinaire, 2 à 4 si une $\mathrm{S}$. Atm. est déjà installée.

\subsection{Prévision des S. Sol.}

Le đélai dépend de la saison.

- Une S. Sol d'été peut être prévue avec quelques semaines d'avance si une S. Sol existe déjà (S. Atm. au printemps,

(4) Eventuellement, barrage et réservoir souterrain. 
par exemple) et en supposant un climat à venir moyen ; cette prévision sera infirmée si l'été s'avère pluvieux (rare), confirmée si une S. Atm. s'installe.

- Une S. Sol. de printemps et d'automne peut ètre prévue avec un délai de l'ordre de la semaine, c'est-à-dire à peine meilleur que pour les S. Atm., si une S. Atm. et une S. Sol. existent déjà.

\subsection{Prévision des S. Cou.}

Le délai dépend également de la saison.

- Une S. Cou. d'été est prévisible dès le printemps précédent, si l'hiver a été sec, en supposant un climat à venir moyen ; cette prévision sera infirmée si le climat du printemps est très pluvieux, confirmée si le climat est moyen ou si une S. Atm. se présente.

- Une S. Cou. d'automne est prévisible dès le printemps précédent si l'hiver a été sec, en supposant un printemps moyen, un été non exceptionnellement pluvieux et une S. Atm. d'automne; cette prévision n'est donc pas très sûre, en particulier à cause de l'hypothèse de la S. Atm. d'automne, mais il peut être utile de l'envisager pour prendre des mesures à temps.

- Une S. Cou. d'hiver ne peut être prévue au-delà de la semaine et encore seulement si une $\mathrm{S}$. Cou sévit déjà ; il s'agit en fait de la prolongation d'une S. Cou. d'automne, les pluies tardant à reprendre.

\subsection{Prévision des S. Sou.}

Le délai est pratiquement annuel dans le cas moyen et fréquent des nappes que nous avons qualifiées d"ordinaires".

A la sortie d'un hiver avec S. Atm., on peut prévoir une S. Sou. pour toute l'année à venir ; elle ne serait infirmée que par un printemps exceptionnellement pluvieux et raccourcie par un automne exceptionnellement pluvieux.

En phase de S. Sou. sévère, la prévision peut s'étendre à plus d'une année car un climat moyen à venir ne peut atténuer que progressivement une S. Sou. qui résulterait ellemême d'une succession d'années sèches avec, en particulier, des hivers à S. Atm.

S’il fallait résumer les conditions de prévision des $S$. Cou. et des S. Cou. en un seul critère, nous pourrions dire que la pluviosité de l'hiver est décisive et que des prévisions peuvent être faites dès la fin de cette saison pour les quelques mois à venir, avec bien entendu des incertitudes plus ou moins grandes.

\section{Exemples d'application}

Les exemples qui suivent concernent la Région Parisienne.

\subsection{Forte S. Cou. de la fin de 1971.}

Elle a été imprévisible jusqu'à l'été, l'hiver et le printemps ayant été à peu près moyens; dès le milieu de l'été, une S. Atm. nette permettait de prévoir une S. Cou. d'automne en supposant une prolongation de la S. Atm. ; au fur et à mesure de la prolongation de cette S. Atm., la prévision de $\mathrm{S}$. Cou. était confirmée, mais le délai de prévision n'a jamais pu dépasser 1 à 2 semaines, étant donné que des fortes pluies d'automne pouvaient mettre fin assez rapidement à la $\mathrm{S}$. Cou.

\subsection{Tendance à la S. Sou. pendant la période $1970-1975$}

Des la fin de l'hiver 1971, une S. Sou. était naissante. La succession d'hivers plutôt secs et d'automnes avec de franches S. Atm. permettait, dès 1971, de prévoir une prolongation de la $\mathrm{S}$. Sou, au moins jusqu'à l'hiver suivant, voire sur plus d'un an, en supposant des hivers à venir déficitaires ou moyens.

\subsection{Année 1976}

La S. Atm. d'hiver, associée à une S. Sou. résiduelle (cf § 5.2), permettait de prévoir, dès la fin de cet hiver 75-76: - un risque assez élevé de S. Sol. et de S. Cou. vers l'été

- un risque moyen de S. Cou. d'automne, en supposant une forte S. Atm. d'été ou une S. Atm. d'automne - une aggravation de la $\mathrm{S}$. Sou.

La S. Atm. du printemps, imprévisible, confirme et aggrave la prévision précédente :

S. Sol dès le printemps et $\mathrm{S}$. Cou dès la fin du printemps risque sérieux de S. Sol maintenue et de S. Cou. sévères dès l'été, en supposant un climat moyen à venir.

A la date de rédaction de cette note (juillet 1976), nous pouvons préciser ainsi ce qui précède :

-- S. Sol persistant l'été, en supposant un climat moyen : elle serait aggravée avec une S. Atm. qui se maintient, atténuée avec un été pluvieux, supprimée seulement si l'été était exceptionnellement pluvieux;

- S. Cou. s'aggravant pendant l'été, sauf climat exceptionnellement pluvieux;

- risque moyen de S. Cou. forte en automne; risque faible de S. Cou. très forte en automne, en supposant une S. Atm. d'automne ; risque rare, mais à ne pas exclure tout à fait, de $\mathrm{S}$. Cou. catastrophique en supposant que la $\mathrm{S}$. Atm. se maintienne en été et en automne.

Nous pourons conclure que la sécheresse de cette année 1976 sera probablement présente, sous une forme ou sous une autre, jusqu'à la fin de l'année. Seule une succession de saisons pluvieuses serait susceptible de l'effacer, et encore faudrait-il attendre l'hiver pour éliminer tout ou partie de la S. Sou.

Nous rappelons qu'il s'agit là de généralités relatives à des conditions naturelles. L'utilisation des équipements hydrauliques locaux peut modifier ces prévisions, par exemple par transfert d'une sécheresse sur une autre.

\section{Possibilités de prévision quantitative}

Nous avons vu qu'une prévision qualitative locale, pour une unité hydraulique donnée, devait nuancer et adapter les généralités précédentes à l'aide de la connaissance locale 
des diverses réserves d'eau concernées et des milieux qui les contiennent (sol. etc. . ).

Pour passer au stade de la prévision quantitative, il faut de plus des observations quantitatives et sur des durées aussi longues que possible : débits, pluies, pièzométrie, humidité du sol, etc. . . Il faut également des méthodes capables de traiter ces observations mais, aujourd'hui, la plupart des équipes spécialisées dans ces problèmes (hydrologues, hydrogéologues, climatologues) possèdent ces outils de prévision (analyses multivariables, régressions multiples, modèles plus ou moins déterministes, bilan, ETR, tarissements, etc. . .) et certaines en ont une pratique courante.

L'essentiel réside dans la qualité, la quantité et la disponibilité de ces données d'observations. Si nous pouvons affirmer, aujourd'hui, qu'à l'échelle d'une unité hydraulique assez grande (au moins quelques centaines de $\mathrm{km}^{2}$ ) il y a toujours quelques données disponibles, il nous faut toutefois préciser que :

-.- leur disponibilité n'est pas toujours immédiate : données non dépouillées, dispersées dans divers services, etc. .

- leur qualité laisse souvent à désirer, même et surtout si elles sont sur support informatique : erreur de codage, fichier non encore utilisé donc non contrôlé, duplicata erroné, etc. .

- les données piézométriques sont encore irrégulièrement réparties, souvent discontinues et pas toujours homogènes;

- les données d'humidité du sol sont potentiellement nombreuses, mais très hétérogènes, très discontinues et leurs fichiers ne sont pas encore organisés et en tous cas ni structurés ni normalisés.

Il en résulte que la construction d'un moyen de prévision permanente de (s) sécheresse (s) ne peut se faire que localement, pour une unité hydraulique bien précisée, et qu'elle exige une étude particulière, pouvant représenter plusieurs mois de travail pour une équipe entrainée.

Par ailleurs, cette prévision quantitative de (s) sécheresse (s) n'a d'intérêt que si elle est faite à échéance d'au moins quelques semaines, voire de plusieurs mois. Elle ne pourra donc pas prévoir les S. Atm. (voir § 4.1). ni les fins de sécheresse dues au retour de pluies abondantes (voir $\S 2$ ), lesquelles sont aussi imprévisibles que les S. Atm. Elle concernera donc les valeurs plus ou moins minimales à craindre de ces réserves d'eau ; ces valeurs devraient être graduées en probabilité ou au moins situées en date (médiane) d'apparition probable.

En d'autres termes, il s'agira d'une prévision de risque, c'est-à-dire de valeurs plus ou moins extrèmes, la probabilité de réalisation de ce risque étant la plupart du temps faible, sauf prévision à court terme et intérieure aux saisons où les aléas climatiques ont peu d'influence sur les sécheresses : l'été pour les S. Cou., l'été et l'automne pour les S. Sou, etc. .

Bien entendu, une prévision de valeurs plutôt moyennes (valeur la plus probable) est également possible, et même utile en complément à la précédente, mais l'erreur sera forte $\left({ }^{5}\right)$ à cause de l'imprévisibilité du climat et ce d'autant plus qu'il s'agira d'une prévision à long terme incluant des saisons où les aléas climatiques sont influents (automne pour les S. Sol. et S. Cou., hiver pour les S. Sou, etc. .).

Pour toutes ces raisons, un modèle de prévision de sécheresse devrait comporter une partie probabiliste plus ou moins importante, même s'il utilise des procédures déterministes comme par exemple les classiques extrapolations de courbes de tarissement pour les $\mathrm{S}$. Cou. où les intégrations de ces courbes de tarissement pour les S. Sou.,

Les utilisateurs devraient d'ailleurs s'habituer à interpréter toute prévision,y compris celles de la météorologie, en probabilité ; c'est-à-dire ne déclencher les procédures de protection que si le produit "(probabilité du risque) x (importance du risque)" dépasse des seuils, à fixer d'un commun accord entre ces utilisateurs et les services sollicités pour leur venir en aide.

(5) Sauf cas particuliers : bassins glaciaires ou neigeux, très grands bassins, craie, etc... 